\title{
Facial colliculus syndrome due to a Herpes simplex virus infection following Herpes labialis
}

\author{
Muhittin Bodur ${ }^{\ominus}$, Rabia Tütüncü Toker ${ }^{\ominus}$, Abdullah Hakan Özmen ${ }^{\bullet}$, \\ Mehmet Sait Okan ${ }^{\odot}$ \\ Division of Pediatric Neurology, Department of Pediatrics, Uludag University Faculty of Medicine, Bursa, Turkey.
}

\begin{abstract}
Background. The facial colliculus is an elevated area that is formed by fibers from the motor nucleus of the 7 'th cranial nerve as they loop over the abducens nucleus. Clinical signs and symptoms of facial colliculus lesions occur primarily due to injury to the abducens nerve nucleus, the facial nerve fibers around the abducens nucleus, paramedian pontine reticular formation, and the medial longitudinal fasciculus. The etiology of facial colliculus lesions varies by age. While tumors, demyelinating lesions, and viral infections can be involved in young individuals' etiology, vascular ischemia is a common causative factor in older people.
\end{abstract}

Case. In this paper, we present a case of facial colliculus syndrome due to its rare occurrence in a young patient; who developed the signs and symptoms after a herpes infection.

Conclusion. Facial colliculus syndrome is rare and the treatment is based on etiology.

Key words: facial colliculus syndrome, herpes infection, diplopia.

Horizontal eye movements occur as products of internuclear connections in the brain stem. A conjugated ipsilateral and a contralateral gaze is achieved via the interactions among the ipsilateral abducens nucleus, contralateral oculomotor nucleus, white matter tracts, and the extraocular muscles.-3 The pons is the primary center for the control of horizontal eye movements. ${ }^{1}$ The paramedian pontine reticular formation (PPRF) is theinterconnection structure for horizontal eye movements. ${ }^{1,4}$ As a signal from the PPRF is transmitted to the abducens nucleus, it's simultaneously transmitted to the abducens nerve and the contralateral oculomotor nucleus via medial longitudinal fasciculus (MLF). ${ }^{1,2}$ This neural pathway explains how different brainstem lesions can lead to various disorders affecting horizontal eye movements. ${ }^{1}$ Different syndromes have been defined according to the

\footnotetext{
$凶$ Muhittin Bodur

dr_muhittin@hotmail.com
}

Received 16th September 2020, revised 2nd December 2020, accepted 24th December 2020. affected brainstem region. "Eight and a half" syndrome is the rare association of "one and a half" syndrome (conjugated horizontal gaze palsy and internuclear ophthalmoplegia) with ipsilateral fascicular cranial nerve VII palsy.,6 First described in detail by Eggenberger in 1998, it is caused by a selective unilateral lesion of pontine tegmentum involving the 6 'th cranial nerve nuclei, the internuclear fibers of the ipsilateral medial longitudinal fasciculus, and the adjacent facial colliculus. ${ }^{7}$ The facial colliculus is an anatomical name given to the elevated area formed by the nucleus of the abducens nerve and the looping around facial nerve fibers in the intrapontine area. Clinical signs and symptoms of facial colliculus lesions ("Eight and a half" syndrome) occur mainly due to injury to the abducens nerve nucleus, the facial nerve fibers around the abducens nucleus, PPRF, and MLF. ${ }^{8}$ In this paper, we present a case with "Eight and a half" syndrome due to a facial colliculus lesion in a young patient who developed the signs and symptoms after a herpes infection; because of its rare occurrence. 


\section{Case Report}

A 12-year-old and 9-month-old girl, known to be healthy, developed a herpetic rash on the lip about a week prior to her admission to our clinic, two days after the emergence of the herpetic rash, redness, and swelling developed around the right eye (Fig. 1). Oral acyclovir and topical acyclovir cream were prescribed to the patient at another medical center. The patient presented to our clinic with diplopia starting over the last two days in addition to her other complaints described above. The medical history of the patient was non-specific. The family history revealed consanguinity between her parents. The patient's physical and neurological examination revealed a bilateral inward gaze restriction during conjugate horizontal gaze (Fig. 1), outward gaze restriction in the right eye, nystagmus and double vision with the upward gaze, and bilateral peripheral facial paralysis being more prominently on the right. The examination of other body systems revealed normal findings.

The laboratory examinations revealed average results for the complete blood count, blood biochemistry, protein $\mathrm{C}, \mathrm{S}$, antithrombin 3, anticardiolipin antibodies, antinuclear antibodies, anti dsDNA, and homocysteine. HSV-1 IgM serology was negative, and HSV1 IgG serology was positive in the blood samples. In the direct examination of the lumbar puncture specimen, 416 erythrocytes

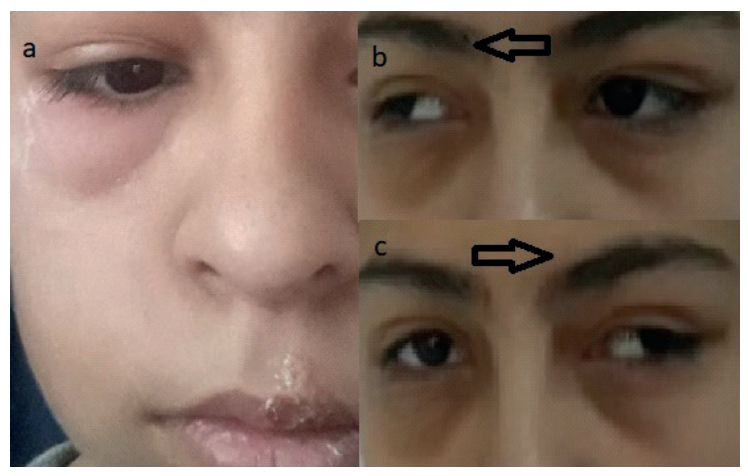

Fig. 1. a. Herpetic eruptions of the case. b-c. Bilateral inward gaze restriction during conjugate horizontal gaze. were counted, no leukocytes were observed. The biochemistry tests of the cerebrospinal fluid (CSF) samples were normal. CSF samples were sent for polymerase chain reaction (PCR) testing for herpes simplex virus (HSV), and the PCR result was positive for HSV-1. The cranial magnetic resonance imaging (MRI) revealed a signal increase in an area of almost $3.5 \mathrm{~mm}$ in diameter on the T2A and FLAIR images at the facial colliculus level in the pons posteriorly. The contrast-enhanced MRI showed mild contrast uptake in this region (Fig. 2). The orbital, spinal, and cranial magnetic resonance angiography (arteriovenous) findings were normal. The diagnosis of facial colliculus syndrome secondary to a herpes infection was considered based on the present results. Treatment with intravenous acyclovir $(10 \mathrm{mg} / \mathrm{kg}$ three times per day) was initiated. Diplopia regressed on the 2nd day of the acyclovir therapy, and facial paralysis reverted on the third day of the treatment. The lateral gaze paralysis regressed on the 10th day of the treatment. After two weeks of intravenous acyclovir treatment, the patient received another week of oral acyclovir treatment. Informed consent was obtained from the family.

\section{Discussion}

The facial colliculus is the anatomical name given to the elevated area formed by the abducens nerve's nucleus and the surrounding facial nerve fibers in the intrapontine area. Clinical signs and symptoms of facial colliculus lesions occur primarily due to injury to the abducens nerve nucleus, the facial nerve around the abducens nucleus, PPRF, and MLF. "Eight and a half" syndrome is the rare association of "one and a half" syndrome (conjugated horizontal gaze palsy [the "one"] and internuclear ophthalmoplegia [the "half"]), with ipsilateral fascicular cranial nerve VII palsy. ${ }^{5,6}$ First described in detail by Eggenberger in 1998, it is caused by a selective unilateral lesion of pontine tegmentum involving the cranial nerve VI nuclei, the internuclear fibers of the ipsilateral medial longitudinal 


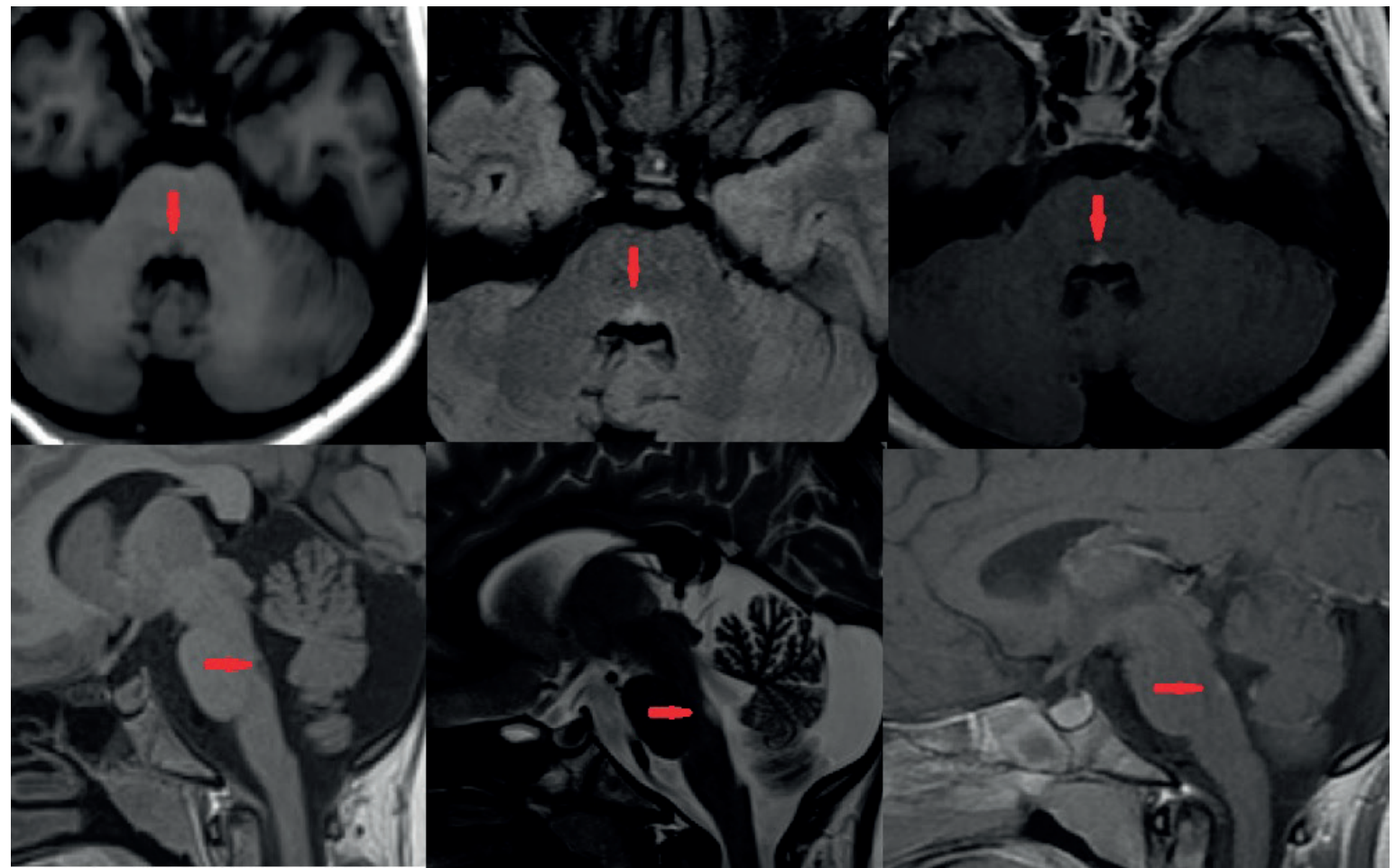

Fig. 2. Cranial MRI: The signal increase in an area of approximately $3.5 \mathrm{~mm}$ was observed in the T2A and FLAIR images at the colliculus facialis level at the pons posterior level.

fasciculus, and the adjacent facial colliculus. Facial colliculus lesions involve the facial nerve, resulting in facial paralysis affecting both the face's upper and lower sides. This condition can often be misdiagnosed as Bell's palsy, an idiopathic cause of peripheral facial paralysis. The abducens nerve innervates the ipsilateral lateral rectus muscle and provides an outward gaze on the same side. It also connects with the contralateral oculomotor nucleus via MLF to achieve control of the contralateral eye's conjugated inward gaze. Therefore, any pathology of the abducens nucleus and PPRF impairs theipsilateral eye's outward gaze and the conjugated inward gaze of the contralateral eye. This situation is different from abducens nerve lesions manifested by limitations in abduction only in the ipsilateral eye. The etiology of facial colliculus lesions varies by age. While tumors, demyelinating lesions, and viral infections can be involved in young individuals' etiology, vascular ischemia is a common causative factor in older people. ${ }^{8,9}$ Facial colliculus syndrome is a clinical condition characterized by the peripheral paralysis of the ipsilateral facial nerve resulting from a facial colliculus lesion, the paralysis of the lateral rectus muscle on the same side, and commonly a conjugate gaze palsy associated with the paralysis of the contralateral medial rectus muscle. ${ }^{8}$ A conjugate gaze palsy can result from an MLF lesion or the involvement of interneurons traveling toward MLF at the abducens nucleus level. Therefore, a combination of peripheral facial nerve palsy, lateral rectus palsy, and conjugate gaze palsy should suggest a potential insult to the facial colliculus. A cranial MRI is a diagnostic test to observe the precise location of the causative lesion. ${ }^{8,9}$ The treatment of facial colliculus syndrome is based on etiology. ${ }^{10}$ The presenting complaint of double vision in the patient; the neurological examination findings of bilateral inward gaze restriction during conjugated horizontal gaze; cranial MRI finding of the involvement of the posterior pons suggested the facial colliculus syndrome. The herpetic lesions of the lip starting in the previous week and the persistence of these lesions at the time 
of admission to our clinic suggested that the herpes infection could explain the etiology, and this was proved by CSF analysis and serologic studies. Therefore, iv acyclovir therapy was given to the patient for 14 days and oral treatment for seven days. Improvements in the presenting complaints of the patient were observed in the follow-up visits. Facial colliculus syndrome is rare, and the treatment is based on etiology.

\section{Author contribution}

The authors confirm contribution to the paper as follows: study conception and design: $\mathrm{MB}$, RTK, AHÖ, MSO; data collection: MB, RTT, $\mathrm{AHÖ}, \mathrm{MSO}$; analysis and interpretation of results: $\mathrm{MB}, \mathrm{RTT}, \mathrm{AHÖ}, \mathrm{MSO}$; draft manuscript preparation: MB, RTT, AHÖ, MSO.

All authors reviewed the results and approved the final version of the manuscript.

\section{Conflict of interest}

The authors declare no conflict of interest.

\section{REFERENCES}

1. Bae YJ, Kim JH, Choi BS, Jung C, Kim E. Brainstem pathways for horizontal eye movement: pathologic correlation with MR imaging. Radiographics 2013; 33: 47-59.
2. Miller MJ, Mark LP, Ho KC, Haughton VM. Anatomic relationship of the oculomotor nuclear complex and medial longitudinal fasciculus in the midbrain. AJNR Am J Neuroradiol 1997; 18: 111-113.

3. Büttner-Ennever JA, Büttner U. Neuroanatomy of the ocular motor pathways. Baillieres Clin Neurol 1992; 1: 263-287.

4. Sparks DL. The brainstem control of saccadic eye movements. Nat Rev Neurosci 2002; 3: 952-964.

5. Skaat A, Huna-Baron R. Eight-and-a-half syndrome: a rare pontine neuro-ophthalmologic syndrome. Arch Neurol 2012; 69: 934-935.

6. Leigh RJ, Zee DS. The Neurology of Eye Movements. (Contemporary Neurology Seris, 70) (4th ed) New York: Oxford University Press, 2006.

7. Eggenberger E. Eight-and-a-half syndrome: oneand-a-half syndrome plus cranial nerve VII palsy. J Neuroophthalmol 1998; 18: 114-116.

8. Kaur R, Singh P, Kajal KS, Aggarwal S. Facial colliculus syndrome. Chrismed J Health Res 2016; 3: 242-243.

9. Alghamdi S, Ayoub O, Alzahrani N, Algahtani $\mathrm{H}$, Shirah B. Vertebral artery occlusion causing facial colliculus and Opalski Stroke syndromes simultaneously. Neurologist 2018; 23: 100-103.

10. Xue F, Zhang L, Zhang L, Ying Z, Shao O, Ding Y. One-and-a-half syndr ome with its spectrum disorders. Quant Imaging Med Surg 2017; 7: 691-697. 